\title{
Cálculo Fracional: Modelagem em Física dos Solos
}

\author{
José Weberszpil Kamila C. do Nascimento ${ }^{*}$ \\ Depto. de Tecnologias e Linguagens, IM, UFRRJ, \\ 26020-740, Nova Iguaçu, RJ \\ E-mail: josewebe@gmail.com, kamilakcn@gmail.com
}

\section{RESUMO}

Nesta contribuição, utilizamos o cálculo de ordem não inteira, também conhecido como cálculo fracionário $(\mathrm{CF})$ para estudar a física dos solos, em especial o transporte de fluidos. Modelos que envolvem o CF vem se mostrando promissores por permitirem uma melhor descrição da dinâmica do transporte de fluidos através de zonas insaturadas em solos.

O modelo mais utilizado para a descrição do sistema hídrico é realizado através da equação de Richard [2] [1]. No entanto, a modelagem clássica envolve o cálculo de ordem inteira. A generalização dessa equação através do CF foi proposta [4], visando aplicações à descrição do processo de transporte de fluidos em meios porosos.

O CF é uma ferramenta matemática tão antiga quanto o cálculo usual e remonta a Leibniz [6]. Existem varias definições para a derivada fracionária, mas as mais utilizadas são as definições de RiemannLiouville e de Caputo [5]. Nessa primeira abordagem utilizaremos somente a primeira: $D^{\beta} f(x)=$ $D^{n}\left[J^{v} f(x)\right]$, onde $D^{\beta} f(x)$ é a derivada de ordem $\beta$ de $f(x)$, para $x>0, D^{n}$ é a derivada de ordem inteira $n$ e $J^{v}$ a integral de Riemann-Liouville de ordem real $v$

$$
J^{v} f(t)=\frac{1}{\Gamma(v)} \int_{a}^{t}(t-\xi)^{v-1} f(\xi) d \xi
$$

onde $\Gamma(v)$ é uma função Gama definida por: $\Gamma(v)=\int_{0}^{+\infty} e^{-t} t^{v-1} d t$.

Nosso objetivo mais básico aqui é o de estudarmos as consequências do uso da generalização fracionária da equação de Richard, seguindo os trabalhos de Pachepsky [4] e Gerolpymaton [2], em comparação aos modelos clássicos de ordem inteira. Numa etapa subsequente pretendemos realizar o mesmo estudo num contexto de derivadas fracionárias chamadas locais ou fractais [3], utilizando com dados obtidos através de medidas realizadas em solos do Brasil.

A lei de Darcy (originalmente aplicada ao estudo de fluxos em meios saturados) pode ser utilizada para meios insaturados e, a partir da mesma, se pode construir a equação de Richard (ER) clássica [2] como:

$$
\frac{\partial \theta}{\partial t}=\frac{\partial}{\partial x}\left(C(\theta) \frac{\partial \theta}{\partial x}\right)
$$

onde $C(\theta)$ é a difusividade da mistura em função do conteúdo volumétrico de água $\theta$.

Usando a condição inicial: $\theta(0, x)=\left\{\begin{array}{lll}\theta_{1}, & \text { se } & x=0, \\ \theta_{0}, & \text { se } & x>0\end{array}\right.$, podemos reformular a ER clássica como uma equação integral:

$$
\theta(t, x)=\theta(0, x)+\int_{0}^{t} \frac{\partial}{\partial x}\left(C(\theta) \frac{\partial \theta}{\partial x}\right) d t .
$$

* bolsista de Iniciação Científica PIBIC/CNPq 
A ideia básica de generalizar a ER consiste em substituir a derivada de tempo $\frac{\partial}{\partial t}$ por uma derivada fracionária de ordem $\alpha$. Esta substituição sugere escrever uma equação da forma:

$$
D^{\alpha}(\theta)=\frac{\partial}{\partial x}\left(C_{\alpha}(\theta) \frac{\partial \theta}{\partial x}\right)
$$

Substituindo a integral do lado direito por um integrando de ordem $0<\alpha \leq 1$, resulta então na equação de Richard fracionária (ERF):

$$
\theta(t, x)=\theta(0, x)+I_{0}^{\alpha} \frac{\partial}{\partial x}\left(C_{\alpha}(\theta) \frac{\partial \theta}{\partial x}\right) .
$$

Simulações numéricas realizadas por Pachepsky [4] para validar a ERF indicam a presença do efeito memória no fenômeno de transporte de água em solos e que pode ajudar a explicar a dependência de escala (leis de potência) e variabilidade na condutividade hidráulica dos solos, encontrada por pesquisadores que aplicaram a versão clássica da ER.

Os resultados de nossos estudos poderão fornecer condições para a elaborações melhores de programas de gerenciamento de poços, simulações de cenários futuros e em consequência a melhor proteção da qualidade da água subterrânea.

Palavras-chave: Modelagem Matemática, Cálculo Fracional, Física dos Solos, Equação de Richard.

\section{References}

[1] Bonganha, Carlos André et al. Conceitos e Fundamentos da Modelagem Matemática para Gerenciamento de Recursos Hídricos Subterrâneos.Revista Analytica. Araraquara. № 30, agosto/2007, p. 116-120. Agosto/Setembro 2007.

[2] Gerolymatou, E; Vardoulakis, I; Hilfer, R. Modelling infiltration by means of a nonlinear fractional diffusion model. Journal of Physics. Institute of Physics Publishing. 39, (2006), 4104-4110.

[3] Hongguan Sun, Mark M. Meerschaert, Yong Zhang, Jianting Zhu, Wen Chen, A fractal Richards' equation to capture the non-Boltzmann scaling of water transport in unsaturated media, Advances in Water Resources 52 (2013) 292-295

[4] Pachepsky, Yakov; Timlin, Dennis; Rawls, Walter. Generalized Richards equation to simulate water transport in unsaturated soils. Elsevier. Journal of Hydrology. USDA, Beltsville, USA. 272 ,2003), p. 3-13.

[5] Podlubny, Igor. Fractional Diferencial Equations: Mathematics in Science and Egeneering. Vol. 198, Academic Press, San Diego, (1999).

[6] Silva, Vivia Santos. Cálculo Fracional Aplicado à Modelagem de Sistemas Viscoelásticos. Monografia. UFRRJ (2014). 